\title{
An alternative explanation of back-relaxation in ionic polymer metal composites
}

\author{
M. Porfiri ${ }^{1 \mathrm{a}}$, A. Leronni ${ }^{\mathrm{a}, \mathrm{b}}$, L. Bardella ${ }^{\mathrm{b}}$ \\ ${ }^{a}$ Department of Mechanical and Aerospace Engineering, New York University Tandon School of \\ Engineering, 6 MetroTech Center, Brooklyn, NY 11201, USA \\ ${ }^{b}$ Department of Civil, Environmental, Architectural Engineering and Mathematics, University of Brescia, \\ Via Branze 43, 25123, Italy
}

\begin{abstract}
The phenomenon of back-relaxation in ionic polymer metal composites (IPMCs) has attracted the interest of the scientific community for two decades, but its physical origins largely remain elusive. Here, we propose an explanation of this phenomenon based on Maxwell stress. From first principles, we demonstrate that IPMC actuation is controlled by the nonlinear interplay between osmotic and electrostatic phenomena. While osmotic pressure tends to produce a rapid bending toward the anode, Maxwell stress generates a slow relaxation toward the cathode. The relative weight of these phenomena is determined by the applied voltage. At voltage levels comparable to the thermal voltage, IPMC actuation is dominated by osmotic effects. As the applied voltage is increased, Maxwell stress overcomes the osmotic pressure, leading to back-relaxation.
\end{abstract}

Keywords: Actuator, Electrochemistry, Maxwell stress, Poisson-Nernst-Planck

\section{Introduction}

Ionic polymer metal composites (IPMCs) are an emerging class of electroactive materials, which have shown strong potential as soft artificial muscles in a wide range of applications across technology and life science [1]. For example, IPMCs have been utilized as propulsion systems in biologically-inspired robots, implantable heart-assist and compression devices, and tactile displays for virtual reality [2,3]. Many more applications are under way, fueled by advancements in three-dimensional printing of free-form IPMCs $[4,5]$. IPMCs typically consist of an ionomeric membrane, neutralized by mobile cations and plated by conductive electrodes. The application of a voltage difference across the electrodes induces an electric field in the ionomer, which is responsible for the controlled migration of cations [6]. The accumulation and depletion of cations through the ionomer is generally regarded as the key physical driver of IPMC actuation, leading to electrostatic, osmotic, and hydraulic forces [1].

\footnotetext{
${ }^{1}$ Corresponding author: mporfiri@nyu.edu
} 
Perhaps, the least understood and most surprising physical phenomenon associated with IPMC actuation is the so-called "back-relaxation". The phenomenon was likely discovered about twenty years ago on Nafion-based samples immersed in a mixed solution of $\mathrm{Na}_{2} \mathrm{SO}_{4}$ and $\mathrm{H}_{2} \mathrm{SO}_{4}$ by Asaka and colleagues [7], and since then a number of studies have demonstrated its ubiquity across a range of material compositions (cation and solvent type) and experimental conditions (degree of hydration, applied voltage, and in-air versus in-solvent testing) [8, 9, 10, 11, 12, 13]. As described in Asaka et al. [7], "in the case of a step voltage [of $1 \mathrm{~V}]$, the composite quickly[, within $100 \mathrm{~ms}$,] bends to the anode side and bends back to the cathode side gradually[, while still under the electric potential]". Back-relaxation in well saturated Nafion samples was observed for a large number of alkali-metal cations, and it was found to be abolished for large, organic, cations [9] at the expense of weaker and slower actuation. The level of hydration was also shown to play a central role on back-relaxation, whereby the cogent removal of a fraction of the water in the ionomer can be used to reduce the phenomenon [13].

The generally accepted view for the physical mechanisms at the basis of back-relaxation focuses on the slow water flow within the ionomer [14]. Specifically, as cations migrate toward the cathode, not only they rapidly carry water molecules attached to them, but do they drag also loose water that is in excess within the ionomer, similar to the "added mass" effect in fluid mechanics. Once carried toward the cathode, the loose water will slowly diffuse back within the ionomer, causing the IPMC to relax. While we cannot dispute or reaffirm this hypothesis, we have discovered that back-relaxation is predicted by the nonlinear balance between osmotic and electrostatic phenomena during cation migration. We argue that Maxwell stress, often neglected in IPMC models, has a central role in explaining back-relaxation. Our explanation is the direct outcome of a recently proposed physicallybased model [15], which was only implemented for steady-state deformations. By simply analyzing the model in time, we unveil the rapid deformation toward the anode, followed by the slow back-relaxation.

Our hypothesis is motivated by experimental observations $[11,16]$, indicating that: i) replacing the step input with a slow ramp that stabilizes at the same step voltage does not mitigate back-relaxation and ii) reducing the intensity of the step input below $1 \mathrm{~V}$ hinders back-relaxation. Both these observations do not seem to favor the claim that backrelaxation is a manifestation of an added mass effect. The former evidence suggests that back-relaxation is attained under slowly-varying applied voltages, which would abate inertia. The latter evidence points at an explanation of back-relaxation where physical nonlinearities are at the core of the phenomenon, beyond linear processes associated with added mass. The possibility that the origins of back-relaxation are beyond hydraulic effects was also put forward in the technical literature $[10,11,12,17,18,19]$, through a detailed analysis of the ionomer microstructure. These theories are construed upon an ionic cluster model, whose complexity may challenge the physical interpretation of the phenomenon and the calibration of simulation parameters from experimental data.

In contrast, the model proposed by Cha and Porfiri [15] is formulated within a continuum theory of IPMCs, grounded on the thermodynamically rigorous theory proposed by Hong et al. [20] to study polyelectrolyte gels. Electrostatic and osmotic forces are not quantified 
from a microscopic analysis of cluster deformation and dipole-dipole interactions. Instead, they naturally stem from a free energy density, which accounts for mechanical stretching, ion mixing, and electric polarization at a macroscopic level. Our approach is based on a minimalistic treatment of the mechanics and electrochemistry of IPMCs, which deliberately neglects viscoleasticity, solvent flow, redox reactions, steric effects, and diffusion processes at the electrodes to establish a first understanding of the possibility that back-relaxation may be simply caused by Maxwell stress.

\section{Modeling framework}

We start by concisely summarizing the model proposed in Cha and Porfiri [15] in the absence of steric phenomena and diffusion processes at the electrodes. For clarity, we adopt the same notation presented therein, which applies to large deformations. Briefly, we describe the state of the ionomer through the deformation gradient with respect to the initial stressfree configuration $\mathbf{F}$, the concentration of mobile cations per unit undeformed ionomer $C$, and the nominal electric displacement $\tilde{\mathbf{D}}$. The free energy density is expressed as the sum of three contributions

$$
W(\mathbf{F}, \tilde{\mathbf{D}}, C)=W_{\text {mec }}(\mathbf{F})+W_{\text {ion }}(\mathbf{F}, C)+W_{\text {pol }}(\mathbf{F}, \tilde{\mathbf{D}})
$$

where $W_{\text {mec }}$ is the contribution from mechanical stretching of the ionomer, $W_{\text {ion }}$ is associated with mixing of mobile cations and fixed anions, and $W_{\text {pol }}$ is related to electric polarization of the ionomer. We hypothesize that the mechanical response is well approximated by a Saint Venant-Kirchhoff constitutive model, such that

$$
W_{\text {mec }}(\mathbf{F})=\frac{\lambda_{\mathrm{L}}}{2}(\operatorname{tr} \mathbf{L})^{2}+\mu_{\mathrm{L}} \operatorname{tr}\left(\mathbf{L}^{2}\right)
$$

where $\mathbf{L}=\frac{1}{2}\left(\mathbf{F}^{\mathrm{T}} \mathbf{F}-\mathbf{I}\right)$ is the Green-Lagrange strain tensor and $\lambda_{\mathrm{L}}$ and $\mu_{\mathrm{L}}$ are Lamé parameters. With respect to ion mixing, we simply consider a solution of two dilute species, such that

$$
W_{\text {ion }}=R T\left[C\left(\ln \frac{C}{C_{0} \operatorname{det} \mathbf{F}}-1\right)-C_{0}\left(\ln \frac{1}{\operatorname{det} \mathbf{F}}-1\right)\right]
$$

where $R=8.314 \mathrm{Jmol}^{-1} \mathrm{~K}^{-1}$ is the universal gas constant, $T$ is the absolute temperature, and $C_{0}$ is the fixed anion concentration. Finally, we assume that the ionomer is dielectrically isotropic, such that

$$
W_{\text {pol }}(\mathbf{F}, \tilde{\mathbf{D}})=\frac{1}{2 \varepsilon} \frac{\mathbf{F}^{\mathrm{T}} \mathbf{F} \cdot \tilde{\mathbf{D}} \otimes \tilde{\mathbf{D}}}{\operatorname{det} \mathbf{F}},
$$

where $\varepsilon$ is the permittivity.

The nominal stress in the ionomer $\mathbf{s}$ is simply computed by taking the derivative of (1) with respect to the deformation gradient, resulting into three distinct contributions, associated with mechanical elasticity $\left(\partial W_{\text {mec }} / \partial \mathbf{F}\right)$, osmotic pressure $\left(\partial W_{\text {ion }} / \partial \mathbf{F}\right)$, and Maxwell stress $\left(\partial W_{\text {pol }} / \partial \mathbf{F}\right)$. Similarly, the nominal electric field is obtained by differentiating (1) with respect to $\tilde{\mathbf{D}}\left(\tilde{\mathbf{E}}=\partial W_{\text {pol }} / \partial \tilde{\mathbf{D}}\right)$, and the electrochemical potential is given by $\mu=$ 
$\mathcal{F} \psi+\partial W_{\text {ion }} / \partial C$, where $\psi$ is the electric potential $(\tilde{\mathbf{E}}=-\nabla \psi)$ and $\mathcal{F}=96485 \mathrm{C} \mathrm{mol}^{-1}$ is the Faraday constant. The last element of the constitutive theory is to ensure that the free energy never increases, which we fulfill by hypothesizing that the cation flux is isotropic with diffusivity $\mathcal{D}$. By using Gauss law, mechanical equilibrium, and mass continuity, one can systematically obtain the governing equations for the ionomer; a detailed derivation is given in the Supplementary Material.

In this study, we focus on a thin IPMC of length $L$ and thickness $2 H \ll L$ undergoing in-plane deformations, such that a beam-like theory applies. By assuming that the curvature $k$ is much smaller than $1 / H$, the electrochemistry is governed by a Poisson-Nernst-Planck (PNP) system along the through-the-thickness coordinate $X$,

$$
\begin{gathered}
-\varepsilon \frac{\partial^{2} \psi(X, t)}{\partial X^{2}}=\mathcal{F}\left[C(X, t)-C_{0}\right] \\
\frac{\partial C(X, t)}{\partial t}=\mathcal{D} \frac{\partial}{\partial X}\left[\frac{\mathcal{F} C(X, t)}{R T} \frac{\partial \psi(X, t)}{\partial X}+\frac{\partial C(X, t)}{\partial X}\right]
\end{gathered}
$$

where $t$ is time. We impose a voltage difference $V$ across the perfectly conducting, ionblocking electrodes (anode at $X=H$ and cathode at $X=-H$ ), such that boundary conditions read

$$
\begin{gathered}
\frac{\mathcal{F} C( \pm H, t)}{R T} \frac{\partial \psi( \pm H, t)}{\partial X}+\frac{\partial C( \pm H, t)}{\partial X}=0 \\
\psi( \pm H, t)= \pm \frac{V(t)}{2}
\end{gathered}
$$

Further, we assume that the ionomer is initially at rest, such that initial conditions read

$$
C(X, 0)=C_{0}, \quad \psi(X, 0)=\frac{V(0)}{2} \frac{X}{H}
$$

From the time evolution of the cation concentration and the electric potential, we compute the bending moments per unit width associated with osmotic pressure, $M_{\text {ion }}$, and Maxwell stress, $M_{\text {pol }}$,

$$
\begin{aligned}
& M_{\mathrm{ion}}(t)=R T \int_{-H}^{H}\left[C(X, t)-C_{0}\right] X \mathrm{~d} X \\
& M_{\mathrm{pol}}(t)=\frac{1}{2} \varepsilon \int_{-H}^{H}\left[\frac{\partial \psi(X, t)}{\partial X}\right]^{2} X \mathrm{~d} X
\end{aligned}
$$

These two moments measure the actuation of the IPMC, and the curvature is ultimately given by

$$
k(t)=-\frac{M_{\mathrm{ion}}(t)+M_{\mathrm{pol}}(t)}{\beta}
$$

where $\beta=(2 / 3) H^{3}\left(\lambda_{\mathrm{L}}+2 \mu_{\mathrm{L}}\right)$ is the bending stiffness.

The PNP system in (5), (6), and (7) neglects the effect of the mechanical deformation on IPMC electrochemistry, whereby the curvature $k$ does not enter the system. The PNP 
system can be solved by following prior work by Porfiri [21] and Wallmersperger et al. [22], which have shown that electric double layers are formed at the ionomer-electrode interfaces, whereas the bulk of the ionomer stays electroneutral. Mobile cations accumulate in the vicinity of the cathode, while depleting the ionomer-anode interface. We propose that the concurrent reshaping of the electric field within the ionomer is responsible for backrelaxation.

In the Supplementary Material, we demonstrate the validity of the PNP system with respect to the so-called modified Poisson-Nernst-Planck (MPNP) system, where mechanical deformations are retained in the nonlinear formulation. Therein, we extend the perturbation analysis presented by Porfiri [21] to study IPMC actuation and shed light on the phenomenon of back-relaxation. A comparison between theoretical predictions from the PNP and MPNP systems is presented, for two different values of the IPMC bending stiffness. Finally, we offer a detailed analysis of the boundary layers' evolution throughout the actuation process, in terms of their relative width, cation concentration, electric potential, and electric field.

\section{Results and discussion}
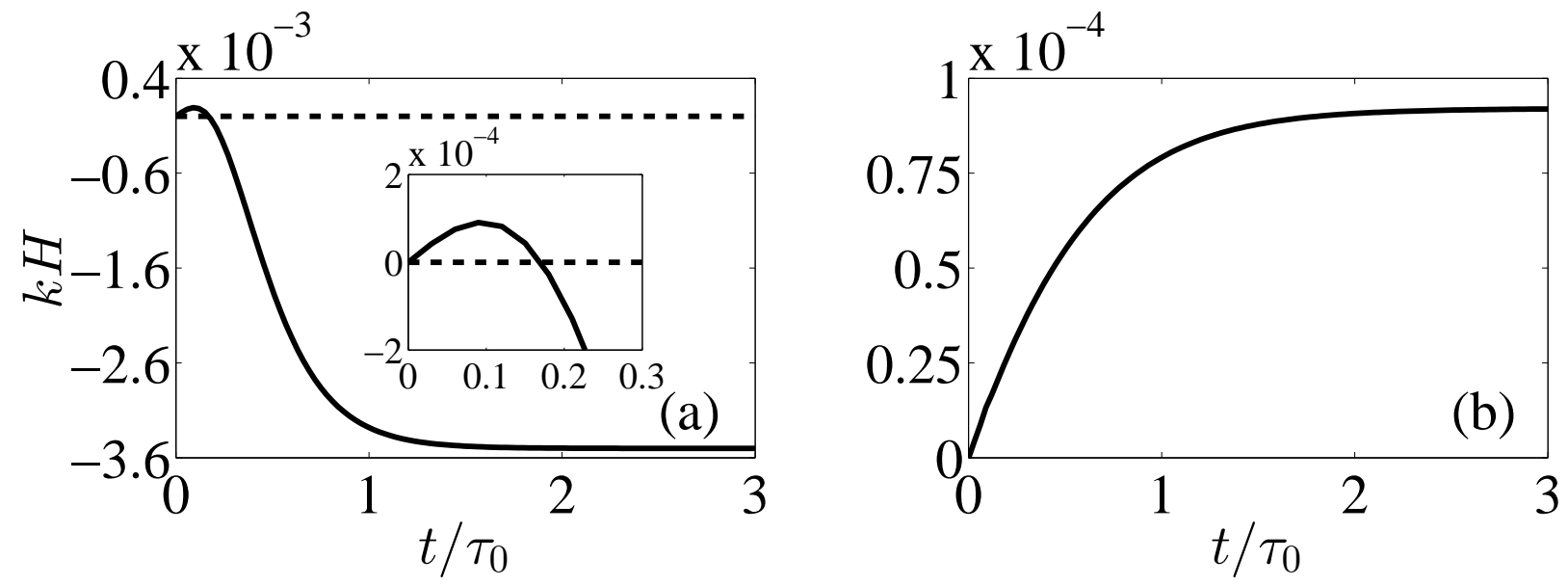

Figure 1: Curvature as a function of time for (a) $V=50 V_{\mathrm{th}}$ and (b) $V=5 V_{\mathrm{th}}$.

Figure 1 shows the curvature of a Nafion-based IPMC for a large and small applied voltages. In agreement with our expectations, for large values of the applied voltage, we observe a strong back-relaxation. Specifically, the IPMC initially bends towards the anode reaching the peak positive curvature $k_{+}$at time $t_{\text {peak }}$, and then slowly relaxes toward the cathode, attaining a negative steady-state curvature $k_{\mathrm{ss}}$. On the other hand, no backrelaxation is noted for a modest voltage input, whereby the IPMC rapidly bends toward the anode and then approaches a positive steady curvature $k_{+}=k_{\mathrm{ss}}$ as time increases. These simulations are conducted for an IPMC at room temperature, $T=300 \mathrm{~K}$, with $H=100 \mu \mathrm{m}$, $C_{0}=1200 \mathrm{~mol} \mathrm{~m}^{-3}, \mathcal{D}=10^{-11} \mathrm{~m}^{2} \mathrm{~s}^{-1}, \varepsilon=4.48 \times 10^{-5} \mathrm{~F} \mathrm{~m}^{-1}$, and $\beta=1 \times 10^{-4} \mathrm{~N} \mathrm{~m}$. 
The values of the constitutive parameters are selected from the relatively wide ranges found in the technical literature $[15,22,23]$ on Nafion-based IPMCs, to obtain round values for both the Debye screening length $\lambda_{\text {Debye }}=1 / \mathcal{F} \sqrt{\varepsilon R T / C_{0}}$ and the characteristic time $\tau_{0}=\lambda_{\text {Debye }} H / \mathcal{D}$, which are equal to $100 \mathrm{~nm}$ and $1 \mathrm{~s}$, respectively. These values should be considered as effective properties of the ionomer, partially accounting for the heterogeneities in physical properties close to the metal electrodes, where so-called intermediate or composite layers exist $[23,24]$. The same parameters are consistently used in what follows to illustrate our claims; an additional case is presented in the Supplementary Material. For clarity, the curvature is scaled by $1 / H$, the voltage by the thermal voltage $V_{\mathrm{th}}=R T / F=25.9 \mathrm{mV}$, and time by $\tau_{0}$.

Figure 2 synoptically illustrates the physical origins of the back-relaxation observed in Figure 1(a). At the onset of double layers' formation, the moment produced by the osmotic pressure in (8a) will cause the IPMC to bend toward the anode. As time progresses and more cations pile-up at the ionomer-cathode interface, a wider region of the ionomer is depleted of cations in the vicinity of the anode. The increasing thickness of the cationdepleted region will cause a difference in the intensity of the electric fields at the double layers, modulating the moment generated by Maxwell stress in (8b). Due to the nonlinear dependence of $M_{\text {pol }}$ on the electric field, compared to the linear dependence of $M_{\text {ion }}$, for sufficiently large voltages, Maxwell stress will finally dominate osmotic pressure causing the IPMC to exhibit back-relaxation.

Figure 3 clarifies the evolution of the bending moments associated with osmotic pressure and Maxwell stress for both the cases considered in Figure 1. The bending moments are scaled with respect to $M_{0}=C_{0} R T H^{2}=29.9 \mathrm{mN}$, consistent with the other nondimensional quantities. For a large applied voltage, although osmotic pressure is initially stronger, it is ultimately surpassed by Maxwell stress. On the other hand, for a small applied voltage, the Maxwell stress is not sufficiently strong to contrast the osmotic pressure, thereby causing the IPMC to monotonically bend toward the anode.

Figure 4 illustrates the dependence of all the salient parameters of IPMC bending on the applied voltage. Therein, the time scale for the back-relaxation $t_{\text {relax }}$ is defined as the time the IPMC takes to reach $90 \%$ of $k_{\mathrm{ss}}$ from $t_{\text {peak }}$. The peak value of the curvature during the initial stage of the motion is approximately constant for $V>5 V_{\mathrm{th}}$. The steady-state curvature shows a more dramatic dependence on the applied voltage: for $0<V<5 V_{\mathrm{th}}$, $k_{\mathrm{ss}}=k_{+}$since back-relaxation is absent; for $5 V_{\mathrm{th}}<V<10 V_{\mathrm{th}}, 0<k_{\mathrm{ss}}<k_{+}$, suggesting that Maxwell stress is not sufficiently strong to reverse the IPMC curvature; and for $V>10 V_{\mathrm{th}}$, we observe back-relaxation, with $k_{\mathrm{ss}}$ continuously decreasing. The rise time is only defined for $V>5 V_{\mathrm{th}}$, and it decreases as a function of $V$, indicating that bending toward the anode becomes faster as the voltage increases. The relaxation time quickly increases for $5 V_{\text {th }}<V<10 V_{\text {th }}$ and then slowly decreases for larger voltages. When back-relaxation is manifested $\left(V>10 V_{\mathrm{th}}\right)$, we confirm that $t_{\text {peak }} \ll t_{\text {relax }}$.

We note that our analysis suggests that the threshold voltage for back-relaxation occurrence is approximately $10 V_{\mathrm{th}}=259 \mathrm{mV}$, different from [16], where it is indicated that back-relaxation is nearly absent for voltages lower than $1 \mathrm{~V}$. This discrepancy could be ascribed to various physical phenomena that are neglected in our model. Among them, 
we mention the heterogeneities in physical properties close to the metal electrodes, where intermediate or composite layers exist $[23,24]$, and steric effects which become important as the cation concentration increases near the cathode $[25,26]$.

In our model, the time scales of $t_{\text {peak }}$ and $t_{\text {relax }}$ are governed by the characteristic time $\tau_{0}$, employed in the selected nondimensionalization. This parameter is directly proportional to the thickness of the sample and to the Debye screening length. The latter is, in turn, related to the permittivity and fixed anion concentration and describes the extension of the electric double layers at the ionomer-electrode interfaces. Conversely, $\tau_{0}$ decreases with the diffusivity, which is controlled by the size and charge of mobile cations, the structure of the membrane, and the viscosity of the solvent [11]. By recalling that $\tau_{0}=1 \mathrm{~s}$ for the selected material parameters, Figs. 4(c) and (d) suggest that the quick bending toward the anode takes place between 0.1 and $1 \mathrm{~s}$, depending on the applied voltage, while back-relaxation occurs on a time scale that is one order of magnitude slower, similar to the observations by Asaka et al. that motivated this work [7].

Varying the material composition and experimental condition will translate into a change in the cation diffusivity and ionomer permittivity, thereby regulating the time scale of the actuation, in agreement with experimental observations from the work of Nemat-Nasser and colleagues $[9,10,11,12]$. Therein, the authors have documented a relatively wide range of variation for the time scales of the quick bending toward the anode, from less than one tenth of a second to over ten minutes, and the following back-relaxation, from less than one minute to more than two hours, for different instances of actuation of Nafion-based IPMCs.

\section{Conclusions}

In the technical literature, most of the actuation models based on the PNP system put forward a single linear phenomenological relationship for the total bending moment associated with IPMC electrochemistry [27, 28, 29], in which the actuation moment is proportional to $\int_{-H}^{H}\left(C(X, t)-C_{0}\right) X \mathrm{~d} X$ through an experimentally identified coupling constant. As a result, IPMC actuation would monotonically increase in time, proportionally to $M_{\text {ion }}$ in Figure 3, thereby hampering the possibility of capturing back-relaxation.

Compared to these linear models for IPMC actuation, a microscopic analysis of cluster deformation and dipole-dipole interactions yields a richer description of IPMC actuation $[10,11,12,17,18,19]$. Such an analysis allows for isolating osmotic from electrostatic effects and identifying the presence of a nonlinearity in the electrostatic pressure. The latter is responsible for an actuation moment that is proportional to $\int_{-H}^{H} f\left(C(X, t)-C_{0}\right) X \mathrm{~d} X$, where $f$ is some nonlinear function that highlights the role played by differences in the cathode and anode double layers on IPMCs actuation. A similar dependence has been posited in the phenomenological models proposed by Wallmersperger et al. [30] and Wallmersperger et al. [31] toward a more refined description of the bending toward the anode, rather than a specific treatment of back-relaxation.

Different from any of these theories, in our model the nonlinearity is associated with the intensity of the electric field rather than the net charge. While we cannot offer experimental evidence that would support our proposal, we favor a dependency of the bending 
moment on the electric field based on the following reason. Removing Maxwell stress from our thermodynamically-consistent formulation would eliminate the energy associated with dielectric polarization, which in turn would hinder the derivation of the Poisson equation in (5a). Perhaps, a more detailed description of the electrode-ionomer interfaces could help resolve this paradox, by masking the effect of Maxwell stress, without challenging the validity of Poisson equation. Future work should seek to clarify this aspect through detailed two-dimensional simulations [32].

Other important areas of future research should aim at including: i) steric effects associated with the cation pile-up at the ionomer-cathode interface $[15,29,25,26]$; ii) diffusion phenomena within the composite layers, leading to a Warburg impedance [24, 33]; and iii) fluid-structure interactions during actuation, shaping the time response of the IPMC [34]. These phenomena are expected to play a significant role in the quantification of backrelaxation as a function of material composition and experimental conditions, toward feedback design to mitigate back-relaxation [35].

\section{Acknowledgments}

This research was supported by the National Science Foundation under grant \# OISE1545857. A. Leronni thanks the H2CU and University of Brescia for the financial support during his stay at New York University. The authors are thankful to Hesam Sharghi for his help with the bibliographical search and Valentina Volpini for the careful review.

\section{References}

[1] M. Shahinpoor, Ionic Polymer Metal Composites (IPMCs): Smart Multifunctional Materials and Artificial Muscles, Royal Society of Chemistry, 2015.

[2] M. Shahinpoor, K. J. Kim, Ionic polymer-metal composites: IV. Industrial and medical applications, Smart Materials and Structures 14 (1) (2005) 197-214.

[3] F. Carpi, E. Smela, Biomedical Applications of Electroactive Polymer Actuators, Wiley Online Library, 2009.

[4] J. D. Carrico, N. W. Traeden, M. Aureli, K. K. Leang, Fused filament 3d printing of ionic polymer-metal composites (ipmcs), Smart Materials and Structures 24 (12) (2015) 125021.

[5] F. Cellini, A. Grillo, M. Porfiri, Ionic polymer metal composites with polypyrrole-silver electrodes, Applied Physics Letters 106 (13) (2015) 131902.

[6] I.-S. Park, S.-M. Kim, D. Pugal, L. Huang, S.-W. Tam-Chang, K. J. Kim, Visualization of the cation migration in ionic polymer-metal composite under an electric field, Applied Physics Letters 96 (4) (2010) 043301.

[7] K. Asaka, K. Oguro, Y. Nishimura, M. Mizuhata, H. Takenaka, Bending of polyelectrolyte membraneplatinum composites by electric stimuli I. response characteristics to various waveforms, Polymer Journal 27 (4) (1995) 436-440.

[8] Y. Bar-Cohen, X. Bao, S. Sherrit, S. Lih, Characterization of the electromechanical properties of ionomeric polymer-metal composite (IPMC), in: Proceedings of SPIE Smart Structures and Materials Symposium, EAPAD Conference, San Diego, 2002.

[9] S. Nemat-Nasser, Y. Wu, Comparative experimental study of ionic polymer-metal composites with different backbone ionomers and in various cation forms, Journal of Applied Physics 93 (9) (2003) $5255-5267$. 
[10] S. Nemat-Nasser, S. Zamani, Y. Tor, Effect of solvents on the chemical and physical properties of ionic polymer-metal composites, Journal of Applied Physics 99 (10) (2006) 104902.

[11] S. Nemat-Nasser, Y. Wu, Tailoring the actuation of ionic polymer-metal composites, Smart Materials and Structures 15 (4) (2006) 909.

[12] S. Nemat-Nasser, S. Zamani, Modeling of electrochemomechanical response of ionic polymer-metal composites with various solvents, Journal of Applied Physics 100 (6) (2006) 064310.

[13] Z. Zhu, L. Chang, K. Takagi, Y. Wang, H. Chen, D. Li, Water content criterion for relaxation deformation of nafion based ionic polymer metal composites doped with alkali cations, Applied Physics Letters 105 (5) (2014) 054103.

[14] M. Shahinpoor, K. J. Kim, Ionic polymer-metal composites: III. Modeling and simulation as biomimetic sensors, actuators, transducers, and artificial muscles, Smart Materials and Structures 13 (6) (2004) $1362-1388$.

[15] Y. Cha, M. Porfiri, Mechanics and electrochemistry of ionic polymer metal composites, Journal of the Mechanics and Physics of Solids 71 (2014) 156-178.

[16] G. Del Bufalo, L. Placidi, M. Porfiri, A mixture theory framework for modeling mechanical actuation of ionic polymer metal composites, Smart Materials and Structures 17 (4) (2008) 045010.

[17] S. Nemat-Nasser, Micromechanics of actuation of ionic polymer-metal composites, Journal of Applied Physics 92 (5) (2002) 2899-2915.

[18] D. Schicker, T. Wallmersperger, Modeling and simulation of the chemo-electro-mechanical behavior of ionic polymer-metal composites, Journal of Applied Physics 114 (16) (2013) 163709.

[19] Z. Zhu, K. Asaka, L. Chang, K. Takagi, H. Chen, Multiphysics of ionic polymer-metal composite actuator, Journal of Applied Physics 114 (8) (2013) 084902.

[20] W. Hong, X. Zhao, Z. Suo, Large deformation and electrochemistry of polyelectrolyte gels, Journal of the Mechanics and Physics of Solids 58 (4) (2010) 558-577.

[21] M. Porfiri, Charge dynamics in ionic polymer metal composites, Journal of Applied Physics 104 (10) (2008) 104915.

[22] T. Wallmersperger, B. J. Akle, D. J. Leo, B. Kröplin, Electrochemical response in ionic polymer transducers: an experimental and theoretical study, Composites Science and Technology 68 (5) (2008) $1173-1180$.

[23] R. Tiwari, K. J. Kim, Effect of metal diffusion on mechanoelectric property of ionic polymer-metal composite, Applied Physics Letters 97 (2010) 244104.

[24] Y. Cha, M. Aureli, M. Porfiri, A physics-based model of the electrical impedance of ionic polymer metal composites, Journal of Applied Physics 111 (12) (2012) 124901.

[25] M. S. Kilic, M. Z. Bazant, A. Ajdari, Steric effects in the dynamics of electrolytes at large applied voltages. I double-layer charging, Physical Review E 75 (2) (2007) 021502.

[26] M. S. Kilic, M. Z. Bazant, A. Ajdari, Steric effects in the dynamics of electrolytes at large applied voltages. II modified Nernst-Planck equations, Physical Review E 75 (2) (2007) 021503.

[27] S. Nemat-Nasser, J. Y. Li, Electromechanical response of ionic polymer-metal composites, Journal of Applied Physics 87 (7) (2000) 3321-3331.

[28] Z. Chen, D. Hedgepeth, X. Tan, A nonlinear, control-oriented model for ionic polymer-metal composite actuators, Smart Materials and Structures 18 (5) (2009) 055008.

[29] M. Porfiri, Influence of electrode surface roughness and steric effects on the nonlinear electromechanical behavior of ionic polymer metal composites, Physical Review E 79 (4) (2009) 041503.

[30] T. Wallmersperger, D. J. Leo, C. S. Kothera, Transport modeling in ionomeric polymer transducers and its relationship to electromechanical coupling, Journal of Applied Physics 101 (2) (2007) 024912.

[31] D. Pugal, K. J. Kim, A. Aabloo, An explicit physics-based model of ionic polymer-metal composite actuators, Journal of Applied Physics 110 (8) (2011) 084904.

[32] B. J. Akle, W. Habchi, T. Wallmersperger, E. J. Akle, D. J. Leo, High surface area electrodes in ionic polymer transducers: Numerical and experimental investigations of the electro-chemical behavior, Journal of Applied Physics 109 (7) (2011) 074509.

[33] Y. Cha, M. Porfiri, Bias-dependent model of the electrical impedance of ionic polymer-metal composites, 
Physical Review E 87 (2) (2013) 022403.

[34] W. Chae, Y. Cha, S. D. Peterson, M. Porfiri, Flow measurement and thrust estimation of a vibrating ionic polymer metal composite, Smart Materials and Structures 57 (2015) 229-246.

[35] M. Fleming, K. Kim, K. Leang, Mitigating IPMC back relaxation through feedforward and feedback control of patterned electrodes, Smart Materials and Structures 21 (8) (2012) 085002. 


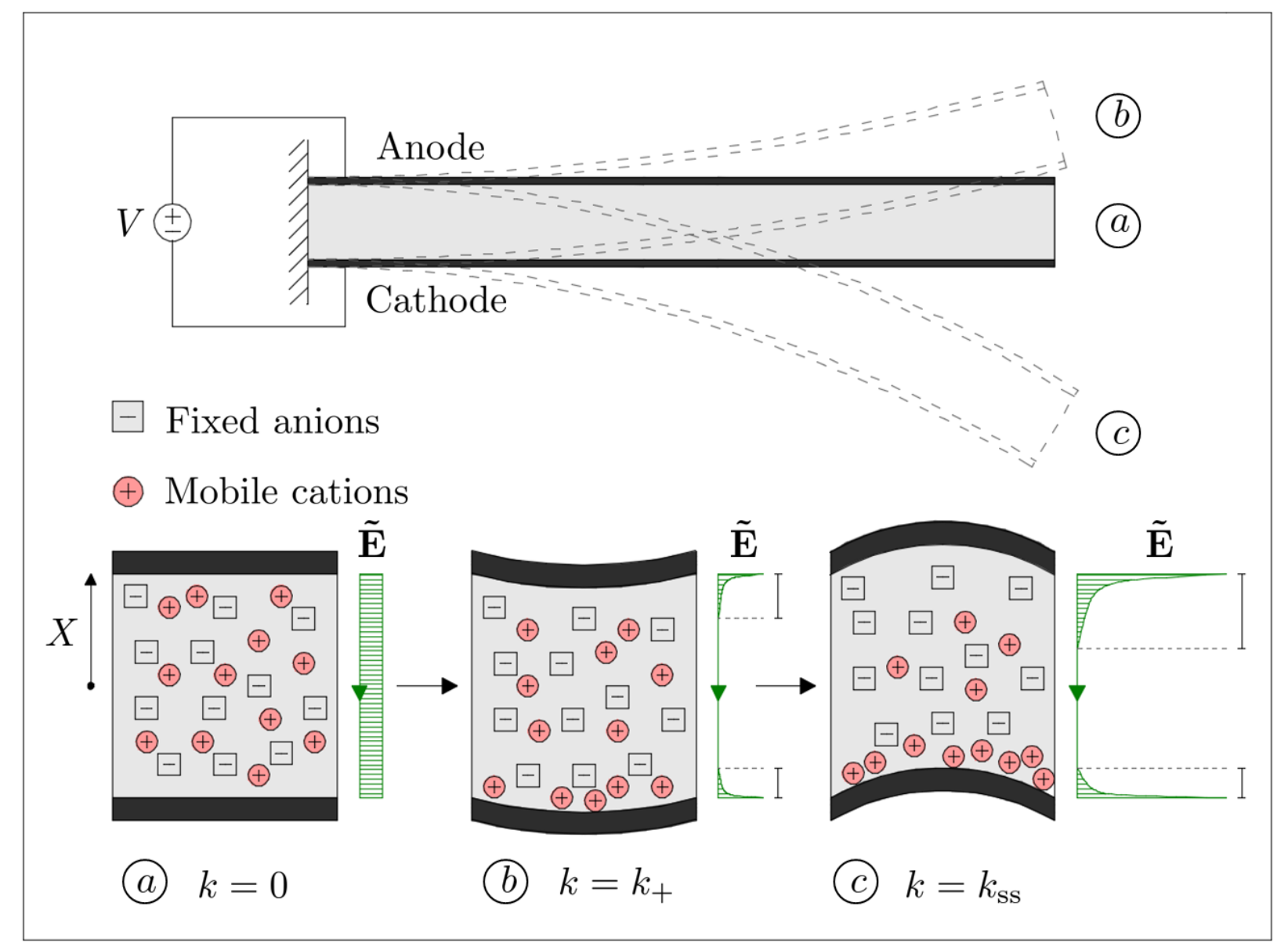

Figure 2: Illustration of IPMC back-relaxation through schematics at three salient instants of the process. These refer to: (a) the initial undeformed configuration, (b) the attained peak positive curvature $k_{+}$, and (c) the steady-state negative curvature $k_{\mathrm{ss}}$. For each instant, we sketch the distribution of cations and the nominal electric displacement $\tilde{\mathbf{E}}$ through the thickness, which control the osmotic pressure and Maxwell stress in the ionomer. The direction of $\tilde{\mathbf{E}}$ is indicated through the green arrows pointing downward and the magnitude along the thickness is depicted as horizontal lines. For clarity, we highlight differences in the thicknesses of the boundary layers, which determine the emergence of a bending moment associated with the Maxwell stress. 

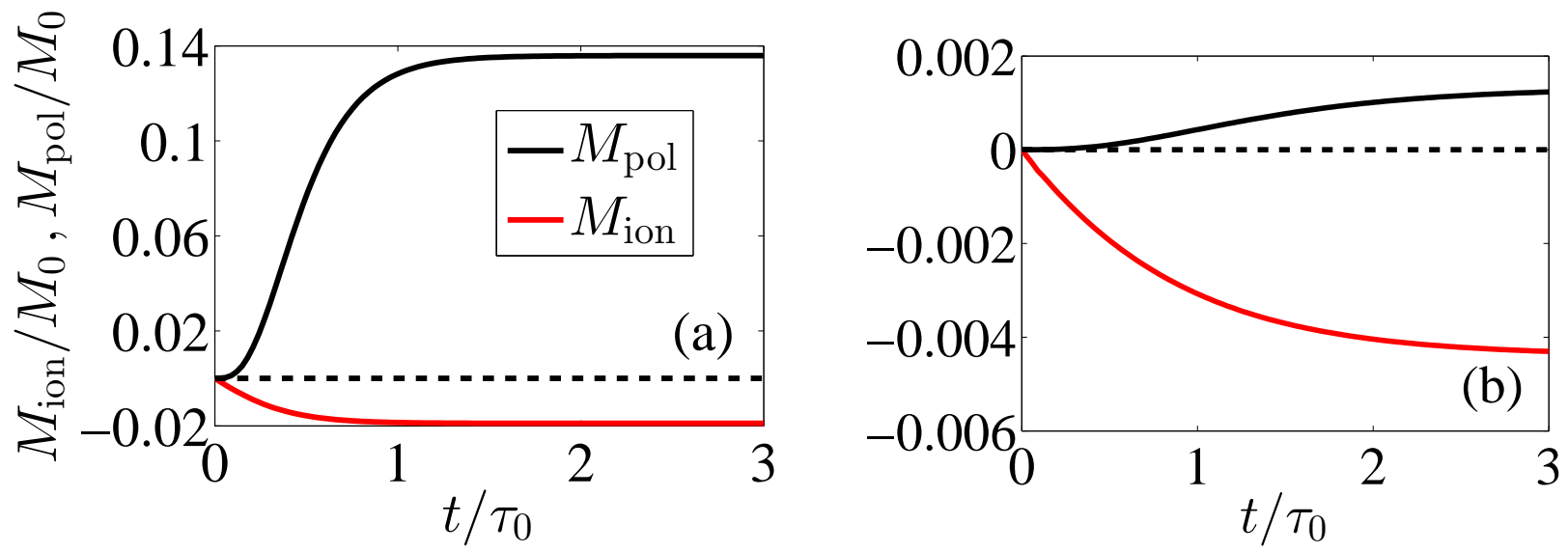

Figure 3: Bending moments due to osmotic pressure and Maxwell stress as a function of time for (a) $V=50 V_{\text {th }}$ and (b) $V=5 V_{\text {th }}$.
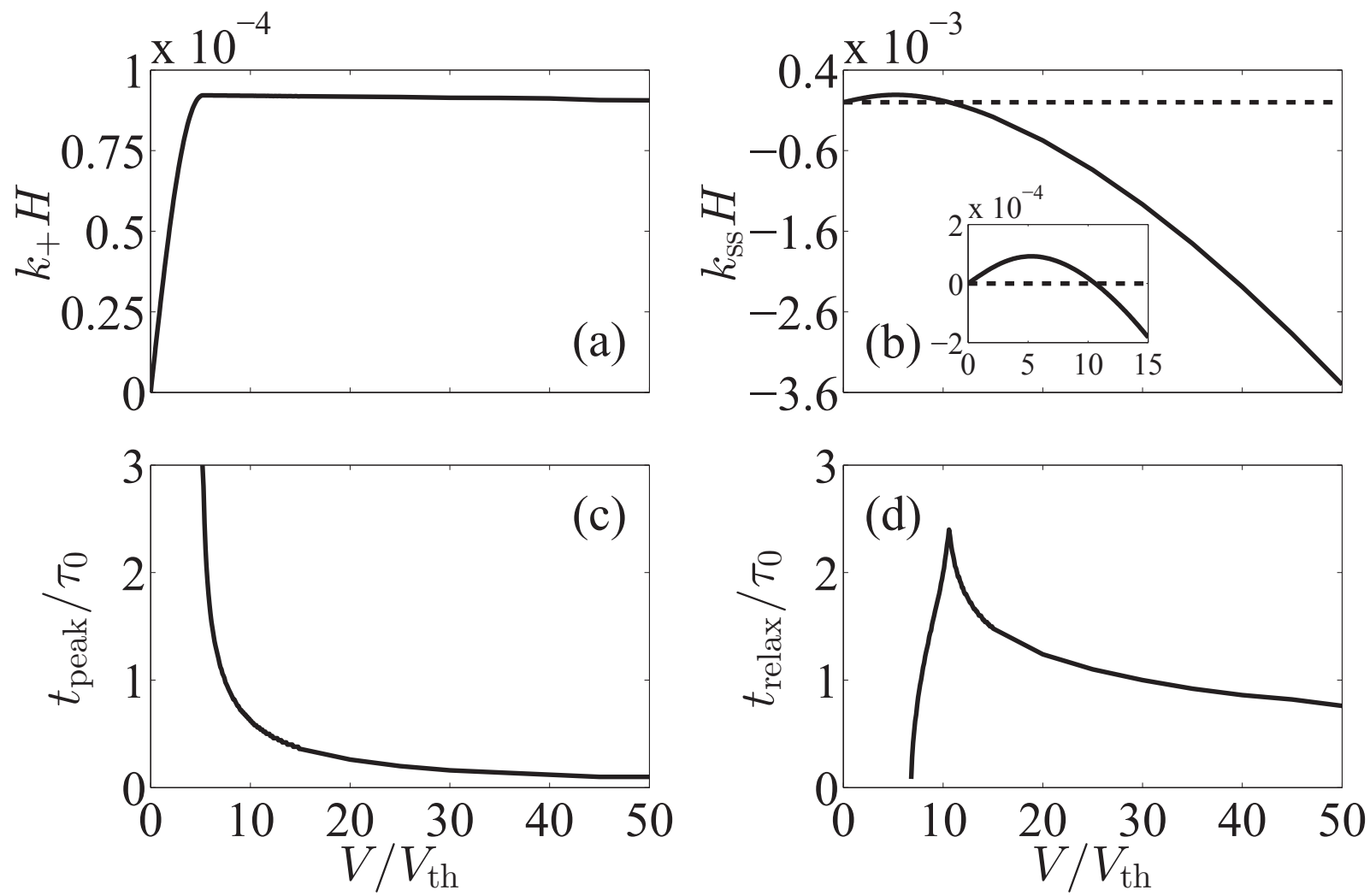

Figure 4: Salient parameters of IPMC bending as a function of $V / V_{\mathrm{th}}$ : (a) peak positive curvature, (b) steady-state curvature, (c) rise time, and (d) relaxation time. 